\title{
An Instinctive Method for Lesion Detection in Diabetic Retinopathy Images using a Novel Spatial Possibilistic C means Clustering in Kernel Space
}

\author{
R.Ravindraiah, S.Chandra Mohan Reddy
}

\begin{abstract}
Diabetic Retinopathy (DR) is the prime cause of vision impediment which originates due micro vascular changes and hyperglycemia stimulated by Diabetes Mellitus (DM). Endothelial lining of the blood capillaries absorbs excess amount of glucose (glycoproteins), hence become thick but are fragile. The swollen capillaries may burst and leak water, proteins, and lipids and tends to fovea expansion. Further it triggers the revascularization to nourish retinal fundus. These new blood capillaries are weak and fragile and can further progress the state to chronic. Pupil dilation for fundus observation leads to many ill effects like head ache, brow pain, blurred vision and light sensitivity. Ophthalmologists cannot administer the pathos well if the symptoms are indolently addressed by the patient and therefore reliability during subjective diagnosis lags. This paper address a qualitative evaluation of novel possibilistic clustering methods with induced spatial constraint in kernel domain to detect the presence of exudates in non-dilated DR images. This methods are compiled in an $N$ dimensional Kernel space which helps to easily segregate the non-linear data regions present in the lower dimensional input space. Also the inclusion of spatial information of a pixel neighborhood will improves the noise handling capability of the proposed methods by easily extricating the noisy pixels from the target lesions and hence improves the system's accuracy in attaining reliable data. Statistical evaluation reveals that the proposed algorithms has attained better sensitivity and specificity compared to existing state-of-art works.
\end{abstract}

Keywords- Diabetic Retinopathy (DR), Exudates, Kernels, Spatial constraint, Fuzzy C means Clustering (FCM) method, Possibilistic C means clustering (PCM) method, Kernel spatial induced Possibilistic methods.

\section{INTRODUCTION}

Diabetic Retinopathy (DR) is a progressive impairment that leads to vision infirmities which is caused due to prolonged history of Diabetes Mellitus. It is rooted by harm to blood vessel within the retinal fundus. It is a treatable ailment if proper care is taken at the earlier stages. Weak and bulged blood capillaries are prime characteristics of DR. In advanced conditions these affected blood capillary walls may rupture, leaking blood and protein based particles into the retinal fundus. These particles are hardened and obstruct the light flow if accumulated at center part of the eye called Macula [1]. Based upon persisted vascular changes in the

Revised Version Manuscript Received on 10 September, 2019.

R.Ravindraiah, Research Scholar, Department of ECE, JNT University Ananthapuramu, Anantapuramu, Andhra Pradesh, India.

(Email: ravindra.ranga@gmail.com)

S.Chandra, Mohan Reddy, Associate Professor, Department of ECE, JNT University Ananthapuramu, Ananthapuramu, Andhra Pradesh, India

(Email:2cmr@gmail.com) retina, DR is classified into i) Non-proliferative (NPDR) and Proliferative (PDR). PDR is due to uncertainty of inconsistent blood vessel growth (neovascularization) in the retina. While NPDR is unassociated with neovascularization. Staid DM consequences may progress NPDR to PDR which tends to severe harm to blood capillaries. Hence in order to nourish retina new blood vessels proliferates. But these abnormal blood capillaries are frail and fragile and make the condition more chronic [2]. Vision threatening cases are 25 times more prevalent in individual with prolonged history of DM. Out of 37 million of total blindness cases, $4.8 \%$ are due DR [3]. And more than $75 \%$ global people with history of DM for above 20 years, have various forms of DR [4]. One in three of working age adults (20 to 65 years) with history of diabetes have some degree of DR and one in ten are expected to develop vision threat. International Association on the Prevention of Blindness (IAPB), assessed that 45 million people are grieved from vision threatening DR, while 145 million people with mild DR in 2015 [5].

Studies of [6] and [7] revealed that about half of 205,000 ophthalmologists world-wide are been contributed by six countries (China, USA, Russia, Japan, Brazil and India). Under developing countries are facing a substantial deficit of ophthalmologists. But in developed countries the figure of practitioners is growing. But this is too insufficient to cope up the subjects as the population $60+$ is rising at twice the rate of ophthalmologists. Hence there is an indispensable need to train eye care professionals to overwhelm this flaring breach amidst need and supply [6]. They marked the ophthalmologists count per million individuals as follows: Less than 1 in 23 countries, 4 in $30 ; 25$ in $48 ; 100$ in 74 countries and Only 18 countries have more than 100 per every million inhabitants. The underdeveloped countries are more suffered as their monetary heights will cause a substantial gap in generation of clinical practitioners. It is expected that by 2030 , there will be only $2 \%$ rise of ophthalmologists compared to $54 \%$ rise of populace with diabetes [7-8]. Further typical dilation process employed by ophthalmologists during initial diagnosis of retinal involves the usage of drops like Mydriatics or Cycloplegic drops These tends to temporary side effects to the victims that lasts for few hours to days [9]. 
Also the patho analysis and screening is truly dependent on the experience and expertise of the clinicians. Thus there is tremendous need to increase Ophthalmologists and enhance the current diagnosis tools. The inclusion of image processing methods into conventional patho analysis system will not only improve the speed of diagnosis but also rises the reliability. Hence the proposed work aims to support the clinicians in quick diagnosis and screening of DR by supporting impending strategies to produce self-reliant investigative setup.

\section{LITERATURE REVIEW}

Currently there exists an increased interest in investigating abnormalities in clinical imaging systems. Multiple works are been proposed to segment or classify retinal abnormalities which make use of various methodologies and are briefed as follows.

Osareh A. [10] et al did a comparative analysis of five classifiers (Linear Delta Rule, K-Nearest Neighbours, neural networks (NN) and quadratic Gaussian classifier) for exudates classification Features from Fuzzy C Means (FCM) are used to feed the classifiers. Using a small dataset of 16 images and attained maximum accuracy of $90.1 \%$ for exudate detection and $90.6 \%$ for OD localization. In [11] they employed FCM for coarse segmentation of fundus images and a genetic algorithm was used to attain features from FCM result and did NN classification. They reported a sensitivity and specificity as $93 \%$ and $94.10 \%$ respectively. Sopharak.A et.al. [12] experimented the classification results of the naïve They attained an overall sensitivity of $93.38 \%$, specificity of $98.14 \%$, accuracy of $98.05 \%$ and precision of $47.51 \%$. Sopharak et. al., [13] applied FCM along with morphological methods on non dilated DR images. They used a local dataset of 40 images and did statistical evaluation using ophthalmologists hand drawn ground truth images and reported an average sensitivity of $92.18 \%$ and specificity of $91.52 \%$. Akram et.al. [14] applied a hybrid fuzzy classifier for to detect bright and dark lesions. They used 290 images taken from DiaretDB0, DiaretDB1, DRIVE and STARE databases and achieved $93.71 \%$ of accuracy. Rocha et al. [15] identified bright and red lesions by constructing a visual word dictionary using and Scale Invariant Feature Transform (SIFT), Speeded up Robust Features (SURF) and k-means clustering. They achieved area under curve (AUC) of 0.88 on DiaretDB1 (89 images) image data set. Zhang et al. [16] evaluated lesion regions using morphological operators. Using classical and contextual features a lesion based classification is done to remove the false positives rom lesion map. They used the public databases like DiaretDB (89 images), e-ophtha (47 images), HEI-MED (169 images) and Messidor (1200 images) and reported AUC values as 0.93, $0.95,0.94$ and 0.90 respectively. Harangi.B et.al.[17] proposed an complex active counter model and various preprocessing steps for feature extraction. Used Baye's classifier and did image level and lesion level classification and achieved image level sensitivity and specificity values as 0.92 and 0.68 (on DIARETDB1 database) and 0.87 and 0.86 (on HEI-MED database). Using lesion level they achieved a sensitivity of 0.86 on DIARETDB1 database. Shuang Yu et. al. [18] addressed a deep learning method to perform pixel bayes classifier by increasing the number of features in steps.

level exudates detection using morphological opening. Then classified the candidate lesions using convolutional neural networks (CNN) on e-Optha (47/82 DR images) dataset and achieved $88.85 \%$ of sensitivity, $96 \%$ specificity and $91.2 \%$ of accuracy respectively. Silkar. S et. al. [19] discriminated bright and dark lesions using a Differential Evolution method and morphological techniques. Using $50 \%$ of images taken from STARE, DRIVE, ROCh and DIARETDB1 databases they reported an average accuracy of $97.71 \%$. In [20] they increased the dataset size to $95 \%$ and attained sensitivity and specificity pair of $94.01 \% / 93.58 \%$ for dark lesion detection and $93.14 \% / 93.19 \%$ for bright lesion detection. Gao Z et.al. [21] developed a DR classification system using a deep CNN. Their model is evaluated, deployed clinically and by employing a four degree classification an average accuracy of $88.72 \%$ is reported on DRIVE, STARE DIARETDB0 and DIARETDB1 datasets.

All these methods didn't employed spatial information of the region of interest which helps to suppress the impact of noisy pixel over the candidate lesions. Moreover these methods are employed in the 2D spatial domain whose performance is purely dependent on the spatial metric employed and possess the chance of carrying the flaws inherent with them. This paper present a novel soft clustering methods performed in $N$ dimensional kernel space with appended spatial weight. Thus the gap present in the existing methods can be made minimal.

\section{METHODOLOGY}

Traditional intensity based FCM (introduced by Dunn [22] and later Bezdek [23] has revised it) and its derivatives isolate spherical and ellipsoidal shaped clusters as they use Euclidean distance metric to measure inter cluster similarity. Likewise different distance metrics are able to elevate only a specific clusters with different profiles. Hence FCM fails to reveal non-spherical structures. Also it robustness lags in the data sets corrupted with noise, outliers and other imaging artefacts. Kernel induced clustering methods persuades a class of vigorous non-Euclidean distance measures in original data space by deriving new objective functions. The original data space centroids and other data samples are mapped non-linearly into an $\boldsymbol{N}$ dimensional feature space, $\boldsymbol{F}$ and carry out clustering. It enhances the conventional clustering algorithm's strength by handling smallest cluster difference, which the direct approach is incapable of, while retaining computational simplicity. But still these methods suffer from noise and outliers as they do not incorporate the evidence of spatial perspective. Pixels of an image are very much correlated to their neighbors and keep nearly similar features. The spatial function is a weighted sum of membership value of pixel under consideration and its neighborhood [24, 25]. Fuzzy C Means (FCM) clustering approach and its derivatives are less immune to noise and outliers as they forces the probabilistic function with equal membership value to every data element. As the data volume increases the problem supplements further. Krishnapuram et.al. [25] addressed Possibilistic C means (PCM) clustering, 
by including a probabilistic constraint, $\eta_{i}$ that relaxes the membership function of FCM. These methods uses a given explicit distance metrics to attain convergence.

\section{PROPOSED METHODS}

\section{A. Kernel Induced Possibilistic C Means (KPCM) clustering algorithm:}

The robustness of traditional PCM in handling hyper volume distribution data set cease as they use Euclidean distance metric which extracts only spherical and ellipsoidal shape clusters. Likewise diverse data profiles are grouped as one by using dissimilar distance metrics. But spatial domain metrics are less resistant to noises and outliers. This problem is suppressed if kernel distance metrics are used and thus improves the robustness of the conventional methods [26, 27]. In this work a single and dual laplacian kernels induced PCM is proposed. In a short form these two methods are represented as L1KPCM (KPCM with single kernel) and L2KPCM (KPCM with dual kernels). Therefore the objective function for Multi kernel induced PCM is given by

$$
J_{M K P C M}=2 \sum_{j=1}^{n} \sum_{i=1}^{C} M_{i j}^{m}\left[1-K\left(x_{j}, c_{i}\right)\right]+
$$

$\sum_{i=1}^{C} \eta_{i} \sum_{j=1}^{n}\left(1-M_{i j}\right)^{m}$

In order to attain the convergence of $\boldsymbol{J}_{\text {MKPCM}}$, the Eq (A.1) is differentiated w.r.t $\boldsymbol{M}_{i j}$ and $\phi\left(\boldsymbol{c}_{i}\right)$ and equate to zero. The resultant $\boldsymbol{M}_{i j}$ and $\phi\left(c_{i}\right)$ that tries to optimize $\boldsymbol{J}_{\boldsymbol{M K P C M}}$ are given by,

$$
\begin{aligned}
& M_{i j}=\left[1+2\left(\frac{1-K\left(x_{j} \cdot c_{i}\right)}{\eta_{i}}\right)^{\frac{1}{m-1}}\right]^{-1} ; \forall i, j \\
& \phi\left(c_{i}\right)=\frac{\sum_{j=i}^{n} M_{i j}^{m} \phi\left(x_{j}\right)}{\sum_{j=1}^{n} M_{i j}^{m}} ; \forall i
\end{aligned}
$$

The evaluation of eq (A.3) is difficult to compute directly. Multiply eq (A.3) with $\phi\left(x_{j}\right)^{T}$ on both sides will revise it as

$$
K\left(x_{j}, c_{i}\right)=\frac{\sum_{j=1}^{n} M_{i k}^{m} K\left(x_{j}, c_{i}\right)}{\sum_{j=1}^{n} M_{i j}^{m}} ; \forall i, j
$$

And $\eta_{i}$ is given by

$$
\eta_{i}=\beta \frac{2 \sum_{j=1}^{n} M_{i j}^{m}\left(1-K\left(x_{j} \cdot c_{i}\right)\right.}{\sum_{j=1}^{n} M_{i j}^{m}} ; \beta>0
$$

\section{Algorithm of the MKPCM:} arbitrarily set the cluster centers $\boldsymbol{C}_{\boldsymbol{i}}$

2. Update membership function, $\boldsymbol{M}_{\boldsymbol{i} \boldsymbol{j}}$ (use equation 2)

3. Calculate $\boldsymbol{K}\left(\boldsymbol{x}_{\boldsymbol{j}}, \boldsymbol{c}_{\boldsymbol{i}}\right)$ (use equation 4)

4. Update the possibilistic constraint $\boldsymbol{\eta}_{i}$ (Use equation 5)

5. Update Objective function $\boldsymbol{J}_{\text {MKPCM }}$ (use equation 1)

Repeat the steps 2 to 5 iteratively until $\left|J^{t}-J^{t-1}\right|<\varepsilon$. Here $\varepsilon$ is minimum value (say 0.0001)

B. Multiple Kernel Induced Spatial Possibilistic C Means (MKSPCM) Clustering algorithm:

As discussed in the earlier section that the incorporation of spatial information into conventional methods will further enhances the noise handling capability of these methods. The proposed MKPCM can be made more robust by appending spatial constraint into it. A Gaussian spatial function, $h_{i j}$ is used in this work, whose mathematical expression is given by

$$
h_{i j}=\sum_{k \in N B_{x_{j}}} \sum_{l \in N B_{x_{j}}} \frac{1}{2 \pi \sigma^{2}} e^{\frac{k^{2}+l^{2}}{2 \sigma^{2}}} M_{k, l}
$$

1. Fix the initial cluster count ' $C$ ' (with $m>0$ ) and

Where $h_{i j}$ is computed over $x_{j}$ and its neighborhood pixels. It represents the belongingness degree of the pixel $x_{j}$ to the $i^{\text {th }}$ cluster in the image with $x_{j}$ as it center. It will be high if the neighborhood belongs to the same sub portion of an image. A neighborhood, $N B_{x_{j}}$ of size $5 \times 5$ centered with $x_{j}$ is taken to compute the $h_{i j}$ value. The spatial function preserves the clustering results by enhancing the original membership. It corrects the misclassified pixels from noisy regions by reducing the weighting of noisy cluster through neighbor pixel labels. The revised membership function, encompassed with spatial function $\boldsymbol{h}_{\boldsymbol{i} \boldsymbol{j}}$ and is given by

$$
M_{i j}^{\prime}=\frac{M_{i j}^{m} h_{i j}}{\sum_{k=1}^{c} M_{i j}^{m} h_{i j}}
$$

The eq (7) is further appended into eq(4) and eq (5) in each iteration step till the objective function reaches a minimum specified value. The objective function with $\boldsymbol{J}_{\text {МкPCM }}$ with induced spatial function can be denoted as $\boldsymbol{J}_{\boldsymbol{M K S P C M}}$

\section{Algorithm of the MKSPCM:}

1. Fix the initial cluster count ' $C$ ' (with $m>0$ ) and arbitrarily set the cluster centers $C_{i}$

2. Update the initial membership function, $\boldsymbol{M}_{\boldsymbol{i} \boldsymbol{j}}$ (use equation 2)

3. Calculate $\boldsymbol{K}\left(\boldsymbol{x}_{\boldsymbol{j}}, \boldsymbol{c}_{\boldsymbol{i}}\right)$ (use equation 4 )

4. Update the possibilistic constraint $\boldsymbol{\eta}_{\boldsymbol{i}}$ (Use equation 5)

5. Compute the spatial function $\boldsymbol{h}_{i j}$ (use equation 6)

6. Update the membership function $\boldsymbol{M}_{\boldsymbol{i j}}$ with induced spatial constraint $\boldsymbol{h}_{i j}$ (use equation 7)

7. Update Objective function $J_{M K S F C M}$ (use equation 1)

Repeat the steps 3 to 7 iteratively until $\left|J^{t}-J^{t-1}\right|<\varepsilon$. Here $\varepsilon$ is minimum value (say 0.0001 )

Single and dual laplacian kernels are induced into MKSPCM

and the statistical evaluation of proposed methods are represented in table 1.

\section{Requirements}

A Personal Computer configured with intel i5 5200 2.20GHz CPU and Raspberry pi 3 Model B with 1GB RAM, quad core $1.2 \mathrm{GHz}$ processor kit are used for simulation of the proposed algorithms. Matlab and Octave 4.0 application softwares are used for simulation purpose. Figure 1 shows the simulation of the proposed methods using Raspberry pi Model B kit.

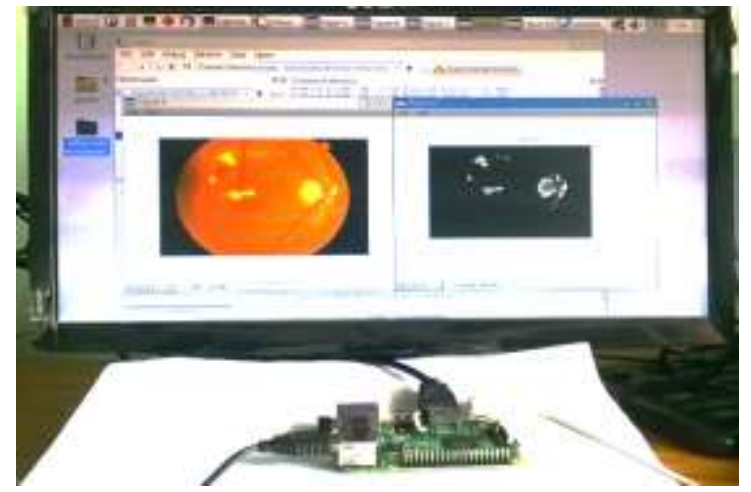

Figure 1. Simulation on Raspberry pi Model B kit. 


\section{RESULTS}

DR is mainly due to internal retinal thickening, preferably near to macula. Hard exudates accumulated over macula are recommended to discover. Exudates are well identified as yellow and white areas characterized with varying shapes, sizes and locations. They are sediments of blood and protein based particles and are usually they appear near to ruptured capillaries. A total of 40 images are used in this work and are selectively taken from five public databases like e-ophtha: A Color Fundus Image Database [28], Diaretdb0 [29],
Diaretdb1 [30], American Society of Retina Specialists [31], High-Resolution Fundus (HRF) Image Database [32] and from private dataset provided by Suthrama Eye Hospital, Madanapalle, A.P, India. Green component of the original fundus image is applied to the proposed methods since it provides more contrast compared to red and blue components. It is then allowed for pre-filtering using median filter for to suppress any form of noises and artefacts and then applied to the proposed methods. The results of the proposed works applied on different databases are as follows:

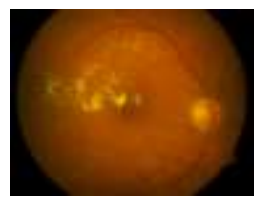

(a)

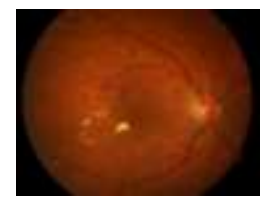

(b)

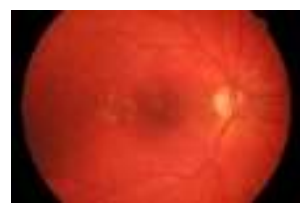

(c)

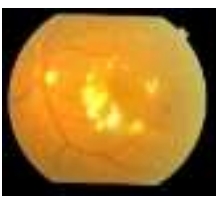

(d)

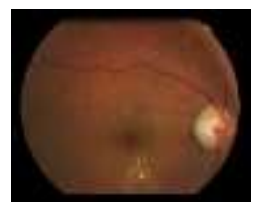

(e)

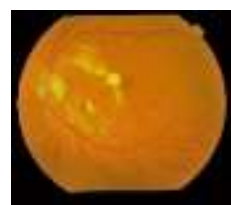

(f)

Figure 2: Typical DR fndus images (a) Image courtesy: Diaretdb0, (b) Image courtesy: Diaretdb1, (c) Image courtesy: High-Resolution Fundus (HRF) databases,(d) Image courtesy: American Society of Retina Specialists, (e) Image courtesy: e-ophtha , (f) Image courtesy: Suthrama Eye Hospital, Madanapalle, A.P, India

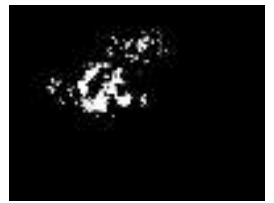

(a)

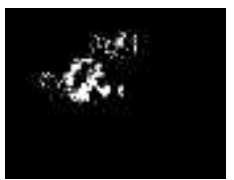

(a)

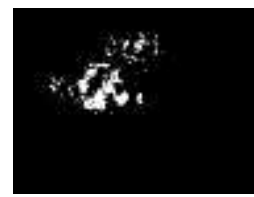

(a)

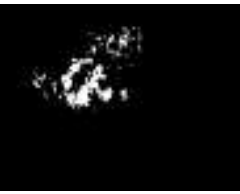

(a)

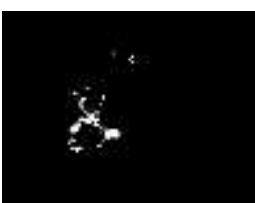

(b)

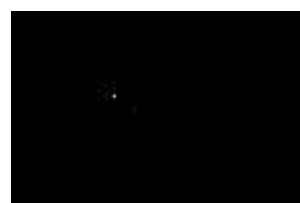

(c)

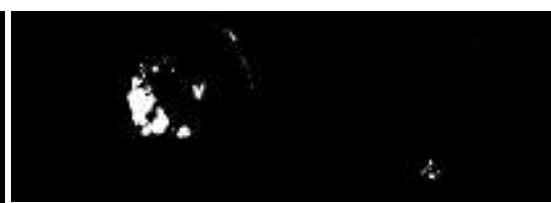

(d)

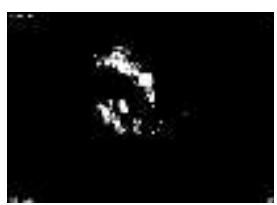

(f)

Figure 3: (a-f) are respective L1KPCM output images of Figure 2: (a-f)

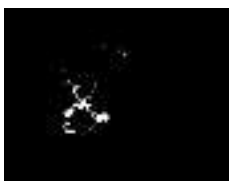

(b)

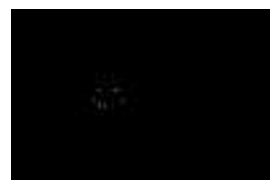

(c)

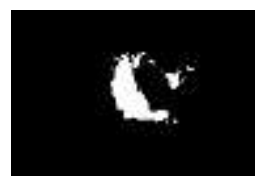

(d)

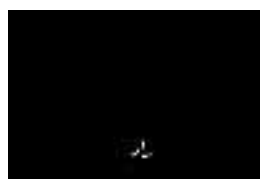

(e)

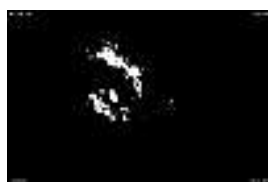

(f)

Figure 4: (a-f) are respective L2KPCM output images of Figure 2: (a-f)

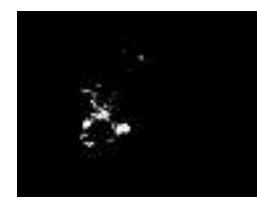

(b)

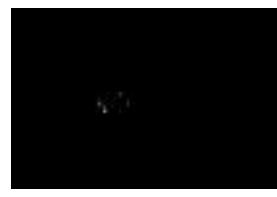

(c)

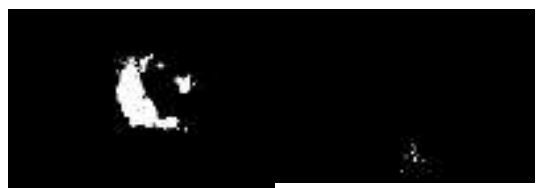

(d) (e)

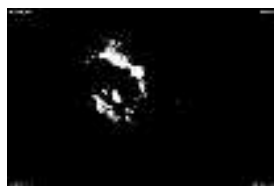

(f)

Figure 5: (a-f) are respective L1KSPCM output images of Figure 2: (a-f)

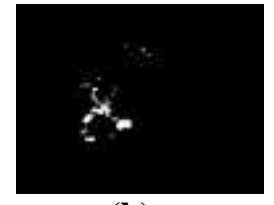

(b)

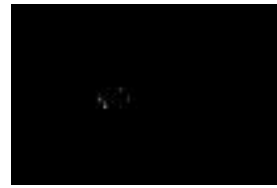

(c)

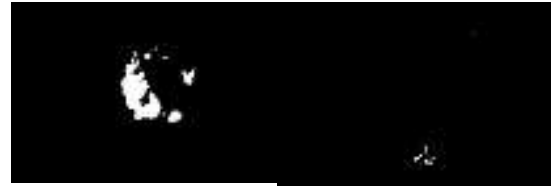

(d) (e)

Figure 6: (a-f) are respective L2KSPCM output images of Figure 2: (a-f)

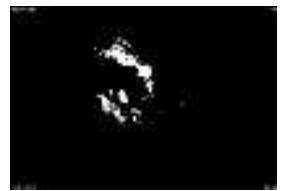

(f)
Figure 2 represent the typical non dilated diabetic retinopathy fundus images taken from five publically available datasets and one from private dataset. They are varied with respect to color, quality, brightness, lesions features, data volume etc. These datasets comprises of DR images ranging from acute to chronic stages. It is observed the unwanted features like blood capillaries are segregated from the target lesions into separate clusters and thus leaving the region of interest onto a unique cluster. Figure $(3-6)$ represents the respective output images of proposed methods in this paper i.e., L1KPCM, L2KPCM, L1KSPCM and L2KSPCM respectively. In order to conserve the segmentation accuracy the optic disc is selectively segregated and then converted into binary version to apply for statistical evaluation.

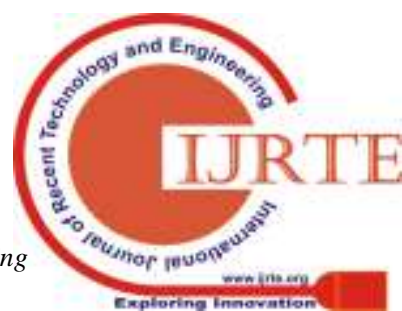


The performance of the methods in detection of abnormal and normal can be evaluated using sensitivity and specificity values. Sensitivity gives the measure of true positives rate and it is a measure of how well the methods detect exudates. While specificity is expedient to detect non-exudates. These values are along with accuracy are assessed using clinical ground truths. The equations of each parameter are as follows.

$$
\begin{aligned}
& \text { Sensitivity }=\frac{T P}{T P+F N} \\
& \text { Specificity }=\frac{T N}{T N+F P} \\
& \text { Accuracy }=\frac{T P+T N}{T P+F P+F N+T N}
\end{aligned}
$$

- True Positive(TP) : Count of correctly classified exudate pixels

- True Negative(TN): Count of correctly classified non-exudate pixels

- False Positive(FP): Misclassified count of non-exudates treated as exudates
- False Negative(FN) : Misclassified count of exudates treated as non-exudates

TABLE 1: STATISTICAL EVALUATION OF THE PROPOSED METHODS

\begin{tabular}{|l|l|l|l|l|}
\hline Sno & $\begin{array}{l}\text { Proposed } \\
\text { Method }\end{array}$ & \% Se* & \% Sp* & \% Acc* \\
\hline 1 & LIKPCM & 90.22 & 99.09 & 94.31 \\
\hline 2 & L2KPCM & 90.12 & 99.18 & 94.01 \\
\hline 3 & L1KSPCM & 93.13 & 99.23 & 95.32 \\
\hline 4 & L2KSPCM & 93.15 & 99.20 & 94.55 \\
\hline
\end{tabular}

Se*: Sensitivity; Sp*: Specificity; Acc*: Accuracy

After the simulation of all the selected images over intel core i5 (4 GB RAM) and raspberry pi model B kit (1 GB RAM) it is observed that the simulation of the proposed methods are taking excess time of around 5.2 times on raspberry kit compared to intel core i5. There exists a need of RAM size improvement and number of cores for handling the multiple threads in parallel.

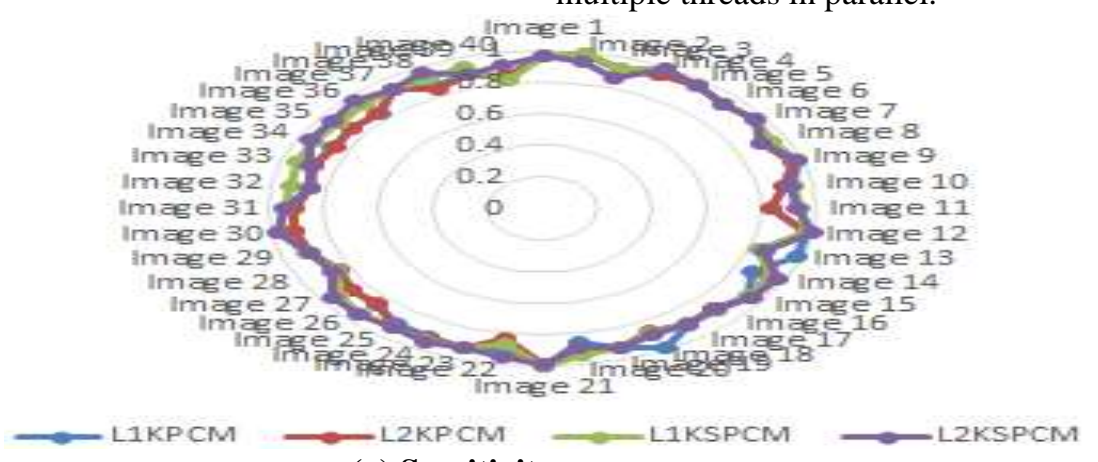

(a) Sensitivity convergence

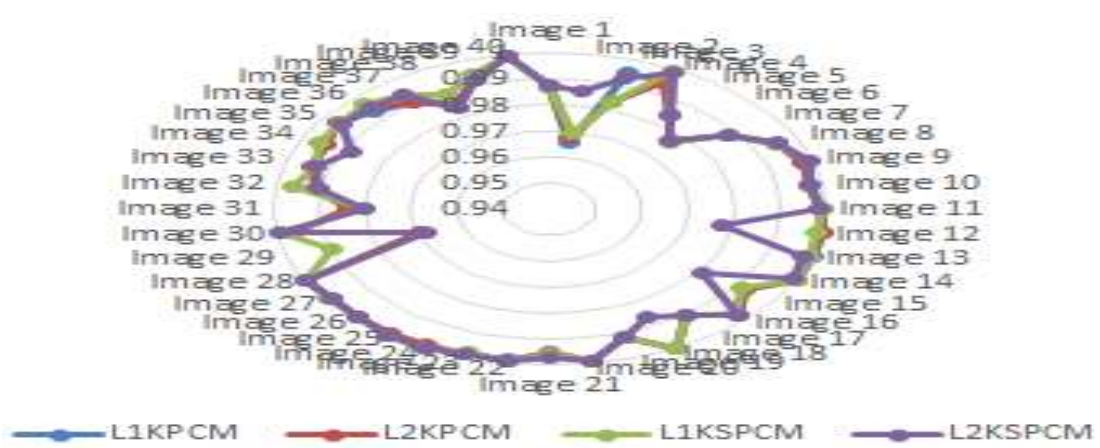

(b) Specificity Convergence

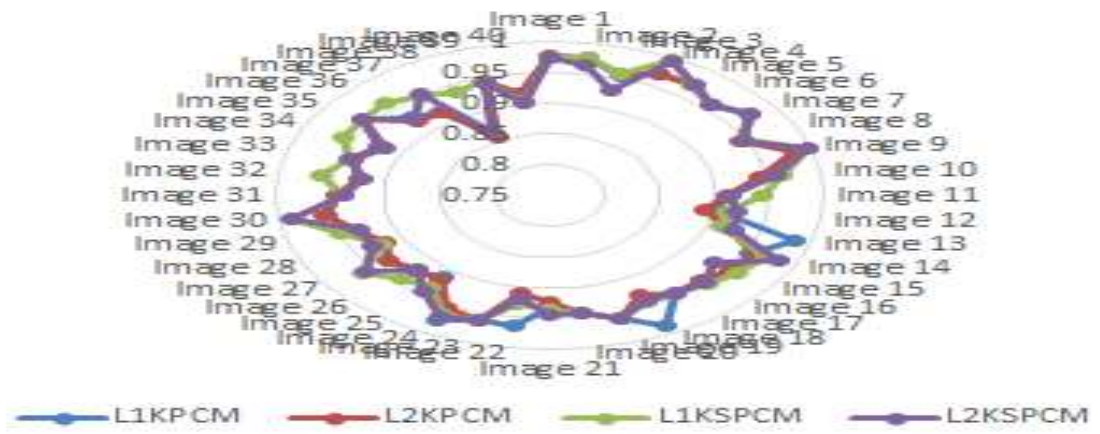

(c) Accuracy Convergence

Figure 7(a-c): Statistical convergence of the proposed methods 


\section{CONCLUSION}

An automatic exudate detection method using a novel and robust clustering methods are presented in this paper. Different versions of multi kernel Possibilistic methods with induced spatial constraint are employed on non-dilated DR fundoscope images. From the experiment results, it can be observed that the proposed methods extract finest details of exudates from non-dilated fundus DR images. Blood vessels are isolated into different clusters and thus there is no scope of any misclassifications from the target lesions. To avoid prominent false alarms, the optic disc is selectively segregated so as to conserve the segmentation accuracy. Also from the statistical metrics reveals optimum result compared to most of the existing works tabulated. The proposed methods do not require strong and heavy preprocessing stages as compared to existing state of art methods. The novelty and robustness of these methods exists with the usage of multiple kernel metrics. Statistical analysis is done using three parameters i.e., sensitivity, specificity and accuracy using clinical ground truths as exemplars. The mean sensitivity, specificity and accuracy of the proposed methods are LIKPCM - 90.22\%, 99.09\%, 94.31\%; L2KPCM 90.12\%, 99.18\%, 94.01\%; L1KSPCM - 93.13\%, 99.23\%, 95.32\%; L2KSPCM - 93.15\%, $99.20 \%$, and $94.55 \%$ respectively. This work can be integrated with ophthalmologists DR diagnosis and screening process through pathology detection and hence can improve the reliability.

\section{ACKNOWLEDGEMENT}

The authors are indebted to Dr. N.Praveen Kanth Reddy, M.B.B.S, M.S (Ophthalmology), Suthrama Eye hospital, Madanapalle, for his esteemed endorsements, which helped to carry out this work successfully.

\section{REFERENCES}

1. The International Agency for the Prevention of Blindness (IAPB). Diabetic Retinopathy. [Online].

2. World Health Organization (WHO), "Prevention of Blindness from Diabetes Mellitus". (2005) [Online]. http://www.who.int/blindness/Prevention of Blindnessfrom Diabetes Mellitus-with-cover-small.pdf.

3. Congdon NG, Friedman DS and Lietman T. Important causes of visual impairment in the world today. JAMA 2003; 290: 2057-60;

4. Fong DS, Aiello L, Gardner TW, et al. Retinopathy in diabetes. Diabetes Care 2004; 27 Suppl 1: S84-87.

5. Nick Kourgialis - Hellen Keller International. Vision Atlas. Available at: http://atlas.iapb.org/ vision-trends/diabetic-retinopathy

6. Serge Resnikoff et. al. The number of ophthalmologists in practice and training worldwide: a growing gap despite more than 200000 practitioners, 2012, British Journal of Opthamology, pp 1-5.

7. International Council of Ophthalmology, "Number of Ophthalmologists in Practice and Training Worldwide" (2010)

8. The International Agency for the Prevention of Blindness (IAPB). Diabetic Retinopathy. [Online]. http://www.iapb.org/vision-2020/what-is-avoidable-blind ness/diabetic-retinopathy

9. Rosas-Romero R, Martinez-Carballido J, HernandezCapistran J, and Uribe-Valencia LJ. A method to assist in the diagnosis of early diabetic retinopathy: Image processing applied to detection of microaneurysms in fundus images, Computerized Medical Imaging and Graphics (44), 2015, pp 41-53

10. A. Osareh, M. Mirmehdi, B. T. Thomas and R. Markham, Classification and localisation of diabetic-related eye disease, in: Proceedings of the 7th European Conference on Computer Vision, LNCS 2353, pp. 502-516, 2002.

11. A. Osareh, M. Mirmehdi, B. Thomas and R. Markham, Automated identification of diabetic retinal exudates in digital colour images, Br J Ophthalmol 87 (2003), pp 1220-1223.

12. A. Sopharak, K. Thet Nwe, Y. A. Moe, M. N. Dailey and B. Uyyanonvara. Automatic exudate detection with a naïve bayes classifier. In International Conference on Embedded Systems and Intelligent Technology (ICESIT), pp 139-142, 2008.

13. Sopharak, B. Uyyanonvara, and S. Barman. Automatic exudate detection for diabetic retinopathy screening, Science Asia, vol. 35, pp. 80-88, 2009. doi: 10.2306/scienceasia1513-1874.2009.35.080

14. M. U. Akram and S. A. Khan. Automated detection of dark and bright lesions in retinal images for early detection of diabetic retinopathy. J. Med. Syst., Vol 36 (5), pp 3151-3162, 2011

15. Rocha, $\mathrm{T}$ et. al., Points of interest and visual dictionaries for automatic retinal lesion detection. Biomedical Engineering, IEEE Transactions on, vol. 59, pp. 2244-2253, 2012.

16. X. Zhang et al. Exudate detection in color retinal images for mass screening of diabetic retinopathy. Medical Image Analysis, vol. 18, pp. 1026-1043.

17. Harangi.B and Hajdu.A. Automatic exudate detection by fusing multiple active contours and region wise classification, Computers in Biology and Medicine 54 (2014) 156-171

18. Shuang $\mathrm{Yu}$, Di Xiao and Yogesan Kanagasingam. Exudate Detection for Diabetic Retinopathy With Convolutional Neural Networks, 2017 IEEE, pp 17441747

19. Sudeshna Sil Kar and Santi P. Maity. Automatic Detection of Retinal Lesions for Screening of Diabetic Retinopathy, IEEE Transactions on Biomedical Engineering, 2018, pp $1-9$

20. Sudeshna Sil Kar and Santi P. Maity. Gradation of diabetic retinopathy on reconstructed image using compressed sensing, IET Journal of Image Processing, 2018, Vol. 12(11), pp. 1956-1963.

21. Zhentao Gaoet.al. Diagnosis of Diabetic Retinopathy Using Deep Neural Networks, IEEE Access, Vol 7, pp $3360-3370,2019$.

22. J.C. Dunn. A fuzzy relative of the ISODATA process and its use in detecting compact well-separated clusters, J.Cybernetics, vol 3, no 3, pp 32-57.

23. J.C.Bezdek. Pattern recognition with fuzzy objective function algorithms, Newyork, Plenum, 1981.

24. R.Ravindraiah and S.Chandra Mohan Reddy. An Automated Exudate detection in Diabetic Retinopathy fundus images using Multi Kernel Spatial Fuzzy C means clustering method, International Journal of Engineering and Technology (UAE), Vol 7 (1.8), pp 10-14, Feb 2018.

25. Raghu Krishnapuram and James M. Kellar. A Possibilistic Approach to Clustering, IEEE Transactions on Fuzzy Systems, Vol 1(2). 1993.

26. R.Ravindraiah, Dr.S.Chandra Mohan Reddy and P.Rajendra Prasad. A Methodical Approach for Segmentation of Diabetic Retinopathy Images" Indian 
Journal of Science and Technology, ISSN: 09745645, 09746846, Vol 9 (S1), pp 1-5, December 2016

27. R.Ravindraiah, Dr.S.Chandra Mohan Reddy and P.Rajendra Prasad. Detection of Exudates in Diabetic Retinopathy Images using Laplacian Kernel induced Spatial FCM Clustering algorithm, Indian Journal of Science and Technology, ISSN: 09745645, 09746846, Vol 9 (15), pp 1-6, April 2016.

28. Decencière E, et al. TeleOphta: Machine learning and image processing methods for teleophthalmology, IRBM (2013), http://dx.doi.org/10.1016/j.irbm.2013.01.010.

29. T. Kauppi V. Kalesnykiene J. K. Kamarainen L. Lensu I. Sorri H. Uusitalo H. Kalviainen and J. Pietila. Diaretdb0: Evaluation database and methodology for diabetic retinopathy algorithms. Technical report, Lappeenranta University of Technology Finland 2006.

30. Kauppi, T., Kalesnykiene, V., Kamarainen, J.K., Lensu, L., Sorri, I., Raninen A., Voutilainen R., Uusitalo, H., Kälviäinen H., and Pietilä, J., DIARETDB1 diabetic retinopathy database and evaluation protocol, In Proc of the 11th Conf. on Medical Image Understanding and Analysis (Aberystwyth, Wales, 2007).

31. ASRS: Advocating for You and Your Patients in 2017, Retina TimesSpring 2017, Vol. 35, No. 1, Issue 68.

32. Budai. A. et.al. Robust Vessel Segmentation in Fundus Images, International Journal of Biomedical Imaging, 2013, pp 1-11 